Applied Physics Report 94-38

\title{
Noise in a Quantum Point Contact due to a Fluctuating Impurity Configuration
}

\author{
J. P. Hessling*, ${ }^{(1)}$ Yu. Galperin, ${ }^{(2)}$ M. Jonson, ${ }^{(1)}$ R. I. Shekhter, ${ }^{(1)}$ and A.M. Zagoskin ${ }^{(1)}$ \\ ${ }^{(1)}$ Department of Applied Physics, Chalmers University of Technology and Göteborg University, S-412 96, Göteborg, Sweden \\ ${ }^{(2)}$ Department of Physics, University of Oslo, P.O. Box 1048 Blindern, N 0316 Oslo 3, Norway, and A.F. Ioffe \\ Physico-Technical Institute, 194021 St. Petersburg, Russia
}

April 26, 2018

\begin{abstract}
We propose a theoretical model for the low-frequency noise observed in a quantum point contact (QPC) electrostatically defined in the 2D electron gas at a GaAs-AlGaAs interface. In such contacts electron scattering by soft impurity- or boundary potentials coherently splits an incoming wave function between different transverse modes. Interference between these modes have been suggested to explain observed non-linearities in the QPC-conductance. In this study we invoke the same mechanism and the time-dependent current due to soft dynamical impurity scattering in order to analyze the low-frequency (telegraph-like) noise which has been observed along with a nonlinear conductance. For the simplified case of a channel with two extended (current carrying) modes, a simple analytical formula for the noise intensity is derived. Generally we have found qualitative similarities between the noise and the square of the transconductance. Nevertheless, incidentally there may be situations when noise is suppressed but transconductance enhanced. In comparison with the more traditional d.c. transport measurements we believe that noise measurements can provide additional information about the dynamical properties of QPCs.
\end{abstract}

PACS numbers: 72.20.-i, 72.70.+m

\section{INTRODUCTION}

During the last several years problems related to electron transport through ballistic point contacts have drawn a lot of attention from the solid state physics community. The most interesting feature of the so-called quantum point contacts (QPC) is the nonlinear character of their current-voltage $(I-V)$ characteristics. This nonlinearity is usually explained within the framework of conductance quantization 1 (for a review see Ref. 5 and references therein). According to this concept the QPC forms a quantum channel that behaves as a wave guide for electrons, the number of transverse modes being dependent both on gate voltage $V_{g}$ and source-drain voltage $V_{s d}$. Consequently, nonlinear features of the $I-V_{s d}$ curve should be observed at driving voltages of the order of the mean spacing between the quantized transverse energy levels; i.e. for $e V_{s d} \approx E_{\mathrm{F}} / N$ [here $E_{\mathrm{F}}$ is the Fermi energy and $N$ the number of modes]. For typical parameters this corresponds to $V_{s d} \approx 1 \mathrm{mV}$. Nevertheless, nonlinear structure in the response has been observed 0 at much lower voltages of order $0.01 \mathrm{mV}$. One could understand such a behavior by taking into account a resonant structure in the conductions steps (see e.g. Refs. 8-11) due to scattering caused by abrupt variations in shape at the entrance and exit regions of the QPC. An explanation

*Electronic address: hessling@fy.chalmers.se appropriate for QPCs with a smooth geometry, which probably corresponds to the experimental situation睡, has been proposed in Ref. 12. It is based upon the concept of coherent mode mixing inside the contact; an electron wave entering the contact is coherently split between different transverse modes due to a scattering potential. During propagation along the channel the electron wave packet becomes deformed because the phase shifts gained by different transverse mode components are not the same. As a result, an interference structure in the current appears because of mode mixing after a second scattering event. Such a behavior is very similar to the well-known electrostatic Aharonov-Bohm effect (see e.g. Ref. 5). It is very important to notice that the described model does not rely upon a short range scattering potential which can produce strong backscattering. On the contrary, the effect persists even if the scattering pgtential is soft, which is the case in ballistic structures 13 . 44 .

The model introduced in Ref. 12 explains the qualitative structure in the measured $I-V$ curves as well as their main quantitative characteristics. We believe that the above mentioned mechanism is responsible for the features observed in Refs. 6 and 7 .

Along with a nonlinear oscillating contribution to the $I-V$ curve of QPCs one has also found a telegraph-like low frequency nois 15 . Our aim here is to analyze noise properties of a QPC within the framework of the model introduced in Ref. 12. Comparison of results on telegraph noise with available experimental data could support (or falsify) the above mentioned model.

The paper is organized as follows. In Section II the 
model employed will be discussed. General analytical expressions for telegraph-like noise, as well as results of numerical calculations, will be given in Section III. In the last Section IV we discuss main results and present our conclusions.

\section{THE MODEL}

Following Ref. 12, we model our QPC - formed in a 2D electron gas by gate electrodes - as an adiabatically smooth channe 16 connecting two equilibrium reservoirs. We assume that the QPC contains two scatterers. The origin of these scattering centers, both taken to be static for the moment, can be the soft potentials formed by one or several of the impurity atoms normally present in QPCs because of doping. The gate electrodes are assumed to provide a hard wall confining potential in the transverse direction, hence creating a channel whose width is furthermore assumed to vary smoothly in the longitudinal direction. The WKB approximation for the electron wave function is therefore applicable. One finds 16

$$
\Psi(x, y, E)=\sum_{n, \pm} a_{n}^{ \pm} \chi_{n}^{ \pm}(x, y, E)
$$

where

$$
\begin{aligned}
\chi_{n}^{ \pm}(x, y, E) & =\sqrt{k_{n, \|}(E, \mp \infty) / k_{m, \|}(E, x)} \phi_{n, x}(y) \\
& \times \exp \left[i \int_{\mp \infty}^{x} d x^{\prime} k_{n, \|}\left(E, x^{\prime}\right)\right] \\
k_{n, \|}(E, x) & =k_{\mathrm{F}} \sqrt{\varepsilon-\varepsilon_{n, \perp}(x)-u g(x)-v s(x)} .
\end{aligned}
$$

Here $E \equiv \varepsilon E_{\mathrm{F}}$ is the total energy of the electron $-E_{\mathrm{F}}$ being the Fermi energy in the leads at zero bias voltage, $V_{s d}=0$ - while $k_{\mathrm{F}}=\sqrt{2 m E_{\mathrm{F}} / \hbar^{2}}$ is the Fermi wave vector and $k_{n, \|}(E, x)$ the longitudinal wave vector along the channel. The transverse part of the wave function, $\phi_{n}(y)$, depends parametrically on the longitudinal coordinate $x$; the corresponding 'transverse' energy eigenvalue is $\varepsilon_{n, \perp}$ and is measured in units of the Fermi energy $E_{\mathrm{F}}$. Hence

$$
\varepsilon_{n, \perp}=\left(\frac{\pi n}{k_{\mathrm{F}} d(x)}\right)^{2},
$$

where $d(x)$ is the coordinate dependent width of the channel. The distribution of the electrostatic potential caused by the applied source-drain voltage is affected by all charges within the contact region; it is described by the dimensionless parameters $v=e V_{s d} / 2 E_{\mathrm{F}}$ and $s(x)$ which appear in the combination $v s(x)$ in (3). In order to match the Fermi levels in the leads we must obviously require that $s( \pm \infty)= \pm 1$. The distribution along the channel for the gate voltage modeled by the dimensionless parameter $u$, is described by the dimensionless function $g(x)$. The plus sign (+) in (2) corresponds to transmission from the left- to the right reservoir, while a minus sign $(-)$ corresponds to transmission in the opposite direction. Without loosing anything essential, we simplify our model by letting $g(x)=1, v=0$ in expression (3) for the WKB wavefunctions.

The interference effects of interest to us are due to coherent scattering by two scatterers. The first one splits an incoming mode into several other modes which then propagate independently. Making use of the unitarity of the scattering matrix one can show 12 . 17 that such a splitting does not change the current at all in the absence of backscattering (if the potential is soft enough). The unitarity condition is simply a statement of conservation of probability, total incoming flux must equal total outgoing flux. The second scatterer makes an additional coherent mode mixing that leads to an interference pattern in the total transmission (or reflection) coefficient, and in the current.

Let us assume that an electron enters from the left, is transmitted after scattering against the left impurity $(L)$, propagates through the contact, passes the right impurity $(R)$ after a second scattering event, and finally escapes the channel on the right hand side. To describe the coherent splitting of the WKB wave function (1) we introduce - following Ref. 12 - for each scatterer a unitary $(2 N \times 2 N)$ scattering matrix,

$$
\hat{S} \equiv\left(\begin{array}{cc}
\hat{r}^{-} & \hat{t}^{+} \\
\hat{t}^{-} & \hat{r}^{+}
\end{array}\right)
$$

such that

$$
\left(\begin{array}{l}
\boldsymbol{\Psi}_{\text {out }}^{+} \\
\boldsymbol{\Psi}_{\text {out }}^{-}
\end{array}\right)=\hat{S}\left(\begin{array}{l}
\boldsymbol{\Psi}_{\text {in }}^{-} \\
\boldsymbol{\Psi}_{\text {in }}^{+}
\end{array}\right)
$$

The $(N \times N)$ submatrices $\hat{r}^{ \pm}$and $\hat{t}^{ \pm}$define the reflection from, and the transmission through the scatterer of the components of the incident wave function. The plus/minus component $\boldsymbol{\Psi}_{\text {in }}^{ \pm}$refers to the incident wave coming from the left/right reservoir, while the plus/minus component $\boldsymbol{\Psi}_{\text {out }}^{ \pm}$refers to the outgoing wave from the scatterer propagating to the right/left. The electron propagation right/left between the scattering events is described by diagonal phase gain matrices $\hat{U}$,

$$
\hat{U}_{i j}^{ \pm}=\delta_{i j} \exp \left[ \pm i \sigma_{j}(E)\right]
$$

where

$$
\sigma_{j}(E)=\int_{x_{L}}^{x_{R}} d x^{\prime} k_{j, \|}\left(E, x^{\prime}\right)
$$

is the phase gained by the $j$-th mode between scatterers $R$ and $L$.

The general expression for the current is 12 ,

$$
\begin{aligned}
I\left(V_{s d}\right) & =\frac{2 e}{h} \int d E \delta n_{\mathrm{F}}(E) \operatorname{Tr}\left[\hat{T}^{+}(E)^{\dagger} \hat{T}^{+}(E)\right] \\
\delta n_{\mathrm{F}}(E) & \equiv n_{\mathrm{F}}\left(E-E_{\mathrm{F}}-e V_{s d} / 2\right) \\
& -n_{\mathrm{F}}\left(E-E_{\mathrm{F}}+e V_{s d} / 2\right)
\end{aligned}
$$


where $\delta n_{\mathrm{F}}$ is the difference between the Fermi-Dirac distribution functions in the reservoirs, and $\hat{T}^{+}(E)$ the total transfer matrix (for transfer from left to right) for a particle with energy $E$, so that $\boldsymbol{\Psi}_{\text {out }}^{+}=\hat{T}^{+} \boldsymbol{\Psi}_{\text {in }}^{+}$. To lowest order, $\hat{T}^{+}=\hat{t}^{+} U^{+} \hat{t}_{L}^{+}$. Making use of Eqs. (5) and (7), one can show 12 that the total current can be expressed as a sum of two parts: $I=I_{\text {diag }}+I_{\text {int }}$. The first part

$$
I_{\mathrm{diag}}=\frac{e}{\pi \hbar} \int d E \delta n_{\mathrm{F}}(E) \sum_{j} R_{j j}(E) L_{j j}(E)
$$

originates from diagonal parts of the matrices $\hat{R} \equiv \hat{t}_{R}^{+\dagger} \hat{t}_{R}^{+}$ and $\hat{L} \equiv \hat{t}_{L}^{+} \hat{t}_{L}^{+\dagger}$. The second contribution,

$$
I_{\mathrm{int}}=\frac{2 e}{h} \int d E \delta n_{\mathrm{F}}(E) \sum_{j<k} 2 \operatorname{Re}\left(R_{j k} L_{k j} e^{i \sigma_{j k}}\right)
$$

where $\sigma_{j k} \equiv \sigma_{j}-\sigma_{k}$, contains all interference effects. In the absence of backscattering Eq. (10) reduces to the usual expression for the current through a ballistic constriction, which exhibits the well known conductance quantization with gate voltage $I_{\text {int }}$ is the contribution from interference between different modes. The expressions (10) and (11) that give the total current were analyzed in Ref. 12. They are the starting points for calculating low-frequency noise.

\section{LOW-FREQUENCY NOISE}

\section{A. General consideration}

One can imagine several mechanisms which lead to low-frequency noise - external, such as low-frequency fluctuations of gate and source-drain voltages, and internal, such as spatial rearrangements of the scattering potentials. In the present study we consider the latter mechanism, which we believe to be the simplest one.

It is well known that there is some disorder in the vicinity of small devices, even if they are of high quality. In any disordered system defects with internal degrees of freedom are present. Interactions with a thermal bath can induce transitions between the corresponding quantum-mechanical states (see Ref. 18 for a review). Usually, such defects switch between two states, leading to a telegraph-like noise pattern. Dynamical defects of this kind have been observed in metal point contacts and tunnel junctions by several authors (see, e.g. Refs. 1924 ) and have been called "elementary fluctuators", or EFs for brevity.

The microscopic structure of the EFs is not completely clear. One of the possible sources of two-state defects is disorder-induced soft atomic vibrations. For low excitation energies the vibrations are strongly anharmonic and can be described as an atom or group of atoms moving in an effective double-well potential. Such entities are known as 25,26 two-level tunneling systems (TLS). They are responsible for the low temperature properties of glassy materials. The generalization of the TLS-model for higher excitation energies has been worked out in Ref. 27. Dynamical defects produce elastic (or electric) fields, slowly varying in time. Conduction electrons are then scattered by these fields. Another possible origin of fluctuations in the scattering potential is electron hopping between adjacent sites in the doped region of the device. The motion of the EFs leads to a variation of the scattering potential in the 2DEG region.

A quantitative theory is not yet completely worked out for any of the two mechanisms mentioned above. In order to describe the main physical picture we use a simplified model; i.e. we assume that one of the scatterers (the left one, say) will maintain its static character, while the other one (right) is allowed to hop between two different spatial positions. The hopping scatterer will from now on be denoted the elementary fluctuator (EF). Its spatial position $x_{R}$ is a random quantity, which we describe as

$$
x_{R}(t)=x_{R}^{0}-\frac{l}{2} \xi(t) .
$$

Here $l$ is the hopping distance; for simplicity, the transverse coordinate has been neglected, leading to an effectively one dimensional description. The random quantity $\xi(t)$ is jumping between the two values \pm 1 at random times, thus describing a telegraph-like process.

The hopping is induced by an interaction with a thermal bath. The transition rate $\Gamma_{+}$from the state $\xi=+1$ to $\xi=-1$ and the reverse rate $\Gamma_{-}$are determined by the nature of hopping and by the interaction between the EF and the thermal bath. From the detailed balance principle we have

$$
\Gamma_{-} / \Gamma_{+}=\exp \left(-\Delta / k_{\mathrm{B}} T\right)
$$

where $T$ is the temperature, and $\Delta$ the energy difference between the states of the EF. Consequently, at high enough temperatures $\left(k_{B} T \gg \Delta\right)$ the hopping rates are almost equal, while at low temperatures there will be a significant difference between the two. The dependence of $\Gamma=\Gamma_{+}+\Gamma_{-}$on $\Delta$ and $T$ is determined by the hopping mechanism (see the discussion in Ref. 18). If the transitions are due to quantum mechanical tunneling $\Gamma \propto \Delta^{k}$ where $k=3$ for EF-phonon interaction and $k=1$ for EF-electron interaction. If transitions are induced by activation on the other hand $\Gamma \propto \exp \left(-W / k_{\mathrm{B} T}\right)$ where $W$ is some activation energy 18 .

We assume that the only effect of the hops made by the scatterer is a variation in the phases $\sigma_{j}$, gained by conducting modes. As has been shown in Ref. 12 the oscillating part of the current is most sensitive to the phase of the modes. Therefore, we expect that the simple model we use contains the most important mechanism for the influence on the current from the EFs. 


\section{B. Analytical expression for noise intensity}

Noise is usually characterized by the current-current correlation function

$$
S(\tau)=\langle I(t+\tau) I(t)\rangle_{t}-\langle I(t)\rangle_{t}^{2}
$$

or by its Fourier transform $S(\omega)$ with respect to time $\tau$. The symbol $\langle\cdots\rangle_{t}$ means average over time $t$ which is (under stationary conditions) just the same as an average over the random process $\xi(t)$ (ergodicity). Because the diagonal part of the current is time-independent, only the interference part enters this expression. Making use of the expression (11) for $I_{\text {int }}$ we obtain the following expression for the current-current correlation function

$$
\begin{aligned}
S(\tau) & =\left(\frac{2 e}{h}\right)^{2} \int d E \delta n_{\mathrm{F}}(E) \int d E^{\prime} \delta n_{\mathrm{F}}\left(E^{\prime}\right) \\
& \times \sum_{j \neq k, l \neq m} M_{j k l m}\left(E, E^{\prime}\right) \Phi_{j k l m}
\end{aligned}
$$

where

$$
\begin{aligned}
M_{j k l m} & =R(E)_{j k} L(E)_{k j} R\left(E^{\prime}\right)_{l m} L\left(E^{\prime}\right)_{m l} \\
\Phi_{j k l m} & =\left\langle e^{i \sigma_{l m}\left(t, E^{\prime}\right)+i \sigma_{j k}(t+\tau, E)}\right\rangle_{t} \\
& -\left\langle e^{i \sigma_{l m}\left(t, E^{\prime}\right)}\right\rangle_{t}\left\langle e^{i \sigma_{j k}(t, E)}\right\rangle_{t} .
\end{aligned}
$$

Assuming that the longitudinal wavevector $k_{j, \|}$ varies slowly as a function of position $x_{R}$ in the channel, one can approximate the phase as

$$
\begin{aligned}
\sigma_{j}(t, E) & \approx \sigma_{j}^{0}(E)+w_{j}(E) \xi(t), \\
w_{j}(E) & \equiv k_{j, \|}\left(E, x_{R}^{0}\right) l / 2 .
\end{aligned}
$$

The superscript 0 indicates that the phase (8), should be evaluated for $x_{R}^{0}$. We then arrive at the following approximate form of the function (16),

$$
\Phi_{j k l m} \approx e^{i\left[\sigma_{j k}^{0}(E)+\sigma_{l m}^{0}\left(E^{\prime}\right)\right]} \mathcal{G}\left[w_{j k}(E), w_{l m}\left(E^{\prime}\right) \mid \tau\right]
$$

where $w_{j i}(E) \equiv w_{j}(E)-w_{i}(E)$, and

$$
\begin{aligned}
\mathcal{G}(x, y \mid \tau) & =K(x, y \mid \tau)-K(x, y \mid \infty), \\
K(x, y \mid \tau) & =\left\langle e^{i x \xi(t+\tau)+i y \xi(t)}\right\rangle_{t} .
\end{aligned}
$$

Note that the function $K(x, y \mid \tau)$ is known 28 as the generating function for the random process $\xi(t)$.

Now, in order to evaluate $K(x, y \mid \tau)$ let us analyze the Master equation for the conditional probability $Q\left(\xi^{\prime}, t^{\prime} \mid \xi, t\right)$ of finding the value $\xi=\xi^{\prime}$ at the time $t^{\prime}$ under the condition $\xi(t)=\xi$. It reads (cf. Ref. 28),

$$
\begin{aligned}
\frac{\partial Q\left(\xi^{\prime}, t^{\prime} \mid \xi, t\right)}{\partial t} & -\xi^{\prime} \Gamma_{+} Q\left(+1, t^{\prime} \mid \xi, t\right) \\
& +\xi^{\prime} \Gamma_{-} Q\left(-1, t^{\prime} \mid \xi, t\right)=0
\end{aligned}
$$

with initial condition $Q\left(\xi^{\prime}, t \mid \xi, t\right)=\delta_{\xi^{\prime}, \xi}$. Taking into account the sum rule

$$
Q\left(+1, t^{\prime} \mid \xi, t\right)+Q\left(-1, t^{\prime} \mid \xi, t\right)=1,
$$

we find the following solution to (20),

$$
\begin{aligned}
Q\left(\xi^{\prime}, t^{\prime} \mid \xi, t\right) & =\frac{1}{2 \Gamma}\left[\Gamma-\xi^{\prime}\left(\Gamma_{+}-\Gamma_{-}\right)+\xi^{\prime} e^{-\Gamma\left|t^{\prime}-t\right|}\right. \\
& \left.\times\left(\Gamma \delta_{+1, \xi}-\Gamma \delta_{-1, \xi}+\Gamma_{+}-\Gamma_{-}\right)\right]
\end{aligned}
$$

The expectation values of all odd and even powers of $\xi(t)$ and its products can now readily be evaluated (since $\xi$ is only allowed to assume the values \pm 1 ). One finds

$$
\begin{aligned}
\left\langle\xi^{2 k}\left(t^{\prime}\right) \xi^{2 n}(t)\right\rangle & =1, \\
\left\langle\xi^{2 k}\left(t^{\prime}\right) \xi^{2 n+1}(t)\right\rangle & =\langle\xi(t)\rangle=\left(\Gamma_{-}-\Gamma_{+}\right) / \Gamma, \\
\left\langle\xi^{2 k+1}\left(t^{\prime}\right) \xi^{2 n+1}(t)\right\rangle & =\left\langle\xi\left(t^{\prime}\right) \xi(t)\right\rangle \equiv C\left(\left|t^{\prime}-t\right|\right) .
\end{aligned}
$$

The function $C \mid\left(t^{\prime}-t \mid\right)$ defined in Eq. (22) can be calculated using its definition, which can be re-expressed as

$$
C\left(t^{\prime}, t\right)=\sum_{\xi, \xi^{\prime}= \pm 1} \xi^{\prime} \xi Q\left(\xi^{\prime}, t^{\prime} \mid \xi, t\right) P(\xi, t) .
$$

$P(\xi, t)$ is here the one-event probability to find the value $\xi$ at time $t$. We find

$$
C\left(t^{\prime}, t\right)=\frac{1}{\Gamma^{2}}\left[\left(\Gamma_{+}-\Gamma_{-}\right)^{2}+4 \Gamma_{+} \Gamma_{-} e^{-\Gamma\left|t^{\prime}-t\right|}\right] .
$$

Expanding the exponential in the generating function (19) and making use of the expectation values (22), together with (24) we obtain,

$$
\begin{aligned}
K(x, y \mid \tau) & =\cos x \cos y \\
& -\sin x \sin y\left[\frac{\left(\Gamma_{+}-\Gamma_{-}\right)^{2}}{\Gamma^{2}}+\frac{4 \Gamma_{+} \Gamma_{-}}{\Gamma^{2}} e^{-\Gamma|\tau|}\right] \\
& -i \frac{\Gamma_{+}-\Gamma_{-}}{\Gamma} \sin (x+y) .
\end{aligned}
$$

For the difference function $\mathcal{G}(x, y \mid \tau)$ defined in (19) we get

$$
\begin{aligned}
G(x, y \mid \tau) & =-\sin x \sin y \frac{4 \Gamma_{+} \Gamma_{-}}{\Gamma^{2}} e^{-\Gamma|\tau|} \\
& =-\frac{\sin x \sin y}{\cosh ^{2}\left(\Delta / 2 k_{\mathrm{B}} T\right)} e^{-\Gamma|\tau|}
\end{aligned}
$$

Here we have used the relation (13) between $\Gamma_{+}$and $\Gamma_{-}$. We see that the quantity $\mathcal{G}\left[w_{j k}(E), w_{l m}\left(E^{\prime}\right) \mid \tau\right]$ in Eq. (18) can be factorized into functions of $E$ and $E^{\prime}$. By their mere construction, the matrices $\hat{R}$ and $\hat{L}$ are Hermitian. Hence one can reduce the double sum as follows,

$$
\begin{aligned}
& \sum_{j \neq k} \sin w_{j k}(E) R(E)_{j, k} L(E)_{k j} e^{i \sigma_{j k}^{0}(E)} \\
& =2 i \sum_{j<k} \sin w_{j k}(E) \operatorname{Im}\left[R_{j k}(E) L_{k j}(E) e^{i \sigma_{j k}^{0}(E)}\right] .
\end{aligned}
$$


Taking the Fourier transform with respect to $\tau$ we find the noise spectrum to be a Lorentzian since the currentcurrent correlation function is exponentially decreasing in time.

Collecting the partial results above, we obtain a general expression for the noise of the form

$$
\begin{aligned}
S(\omega) & =\frac{1}{\cosh ^{2}\left(\Delta / 2 k_{\mathrm{B}} T\right)} \mathcal{L}(\omega) \Lambda^{2}, \\
\mathcal{L}(\omega) & =\frac{1}{\pi} \frac{\Gamma}{\Gamma^{2}+\omega^{2}}
\end{aligned}
$$

where

$$
\begin{aligned}
& \Lambda=\frac{2 e}{\pi \hbar} \int d E \delta n_{F}(E) \\
& \times \sum_{j<k} \sin w_{j k}\left(E, x_{R}^{0}\right) \operatorname{Im}\left[R_{j k}(E) L_{k j}(E) e^{i \sigma_{j, k}^{0}(E)}\right] .
\end{aligned}
$$

The expression (28) is a product of three factors. The first one, $\cosh ^{-2}\left(\Delta / 2 k_{\mathrm{B}} T\right)$, makes it clear that a telegraph-like noise can only appear if the EF can hop due to interactions with a thermal bath. The typical excitation energy is of order $k_{\mathrm{B}} T$. If $\Delta \gg k_{\mathrm{B}} T$ the EF cannot be excited and will remain in its lowest energy state forever. The second factor is a simple Lorentzian function with characteristic width $\Gamma$.

The third factor in (28), $\Lambda^{2}$, contains the same quantities as expression (11) for the interference part of the current. It is of interest to see whether or not the quantity $\Lambda$ can be related to the measured current-voltage characteristics. To address this question we consider the simplified case of a narrow QPC allowing for only two propagating modes. At low temperatures the variation with energy of the matrices $\hat{R}$ and $\hat{L}$ and phases $\sigma$ in an energy interval of order $k_{\mathrm{B}} T$ near the Fermi energy can be neglected. The resulting expressions for the current and for the noise spectrum are in this case very simple,

$$
\begin{aligned}
I_{\mathrm{int}} & =I_{\max } \cos \left[\sigma_{12}^{0}\left(E_{F}\right)+\gamma\right] \\
S(\omega) & =I_{\max }^{2} \frac{\sin ^{2}\left[w_{12}\left(E_{F}\right)\right] \sin ^{2}\left[\sigma_{12}\left(E_{F}\right)+\gamma\right]}{\cosh ^{2}\left(\Delta / 2 k_{\mathrm{B}} T\right)} \mathcal{L}(\omega), \\
I_{\max } & =\frac{4 e}{h} \int d E \delta n_{F}(E)\left|R_{12}(E)_{12} L_{21}(E)\right|
\end{aligned}
$$

The phase difference $\sigma_{12}^{0}\left(E_{\mathrm{F}}\right)$ is of the order of $k_{\mathrm{F}} L$, where $L$ is the spatial separation between the scatterers, $x_{L}-x_{R}^{0}$, and $\gamma$ some phase shift of no interest here. $I_{\max }$ is the maximum value of the interference current with respect to variations in the external parameters. Qualitative conclusions about expressions (30)-(32) will be drawn in Section IV. Next we will present a numerical analysis for the situation with several modes. The role of the channel geometry will also be discussed.

\section{Numerical analysis}

In the case when there are more than two modes propagating through the quantum point contact, one has to rely upon numerical simulations. We have calculated the noise as a function of gate- and source-drain voltages. The quantities usually measured experimentally are the current and the transconductance, defined as

$$
G\left(V_{s d}, V_{g}\right)=\frac{\partial I}{\partial V_{g}}
$$

Only the interference part of the current has been considered because far enough from the conduction steps the diagonal part is practically independent of both gate- and source-drain voltages. The ranges of all parameters were limited in such a way as to give a constant number of propagating modes.

For a numerical evaluation of the general expression (28) for the noise, we need a definite form of $R_{j k}(E) L_{k j}(E)$. This quantity is the product of the two scattering probabilities for scattering from mode $j$ to $k$ by the left scatterer and the analogous probability but time-reversed and with exchanged mode indices for the right scatterer. In a completely symmetric channel (including the impurities) with respect to the coordinate along the channel, $R_{j k}(E) L_{k j}(E)=\left|L_{k j}(E)\right|^{2}$. To simplify the calculations we assume that the only screening of the impurity potential that exists originates from electrons within the gate electrode. This screening can approximately be described by a proper image charge. If the impurity and image charges are separated from the 2DEG by distances $a$ and $b$, respectively, the effective potential is

$$
V(r)=A_{0}\left[\frac{1}{\sqrt{\left(r^{2}+a^{2}\right)}}-\frac{1}{\sqrt{\left(r^{2}+b^{2}\right)}}\right],
$$

where $\mathbf{r}$ is the in-plane coordinate, while $A_{0}$ contains the necessary physical constants.

The scattering probability can be calculated from the Golden rule,

$$
L_{k j}(E)=\frac{2 \pi}{\hbar}|<f| V(x, y)|i>|^{2} \delta\left(E_{f}-E_{i}\right)
$$

where $i$ and $f$ denote the initial and final states.

In the calculation of the matrix element above we have simplified the geometry and regarded the channel to be of constant width. For $r \gg \max (a, b)$, we find that $V(r) \propto$ $1 / r^{3}$. The potential (34) is hence short-ranged, and its influence may be ignored for large $r$. In the opposite limit of small values of $r$, we get $V(r)=A_{0}(1 / a-1 / b)[1-$ $\left.(r / c)^{2}\right]$, where $c=a b / \sqrt{\left(a^{2}+a b+b^{2}\right) / 2}$. The typical range of $V(r)$ is $c$.

Since the expansion for small $r$ of the Lorentzian function $\left[\left(1+(r / c)^{2}\right]^{-1}\right.$ has the same form as the expansion of the actual potential, and since the precise value of $V(r)$ 
for large $r$ is not very crucial as long as it decays quickly enough, we may use the approximation

$$
V(r)=A_{0} \frac{b-a}{a b} \frac{1}{1+(r / c)^{2}} .
$$

The problem of finding $L_{k j}(E)$ can be further simplified if the potential factorizes, so that $V(x, y)=$ $V(x) V(y)$. To get a rough estimate, this factorization can be done as,

$$
V(x, y) \approx A_{0} \frac{b-a}{a b} \frac{1}{\left.1+(x / c)^{2}\right)} \Theta\left[1-(y / c)^{2}\right]
$$

Here we have reduced the $y$ dependence to a step function, but kept the smooth form in the $x$ direction. In this way we prevent the Fourier transform of the potential with respect to $x$ from being oscillatory, which seems unphysical.

Finally, evaluating the matrix element (35) we arrive at the expression

$$
L_{k j}(E)=A \frac{(b-a)^{3} k_{F}}{\left(b^{3}-a^{3}\right) k_{k, \|}} \frac{1-(-1)^{k-j}}{2(k-j)^{2}} e^{-2\left|k_{k, \|}-k_{j, \|}\right| c},
$$

where $A$ contains only fundamental physical constants including an effective dielectric constant. Due to the fact that the potential in the transverse direction is an even function of $y$, we only have a non zero scattering probability between modes of the same parity (i.e. when $k-j$ is an even number).

In Fig. 1 (panel $a$ ) the square of transconductance is plotted versus the dimensionless gate voltage $u=$ $e V_{g} / E_{\mathrm{F}}$. The QPC is assumed to have a parabolic shape, the scatterers being placed symmetrically with respect to the narrowest point. Hence, the width of the channel is

$$
\begin{aligned}
d(x) & =d+(D-d)\left(\frac{x-\bar{x}}{L / 2}\right)^{2}, \\
\bar{x} & =\left(x_{R}^{0}+x_{L}\right) / 2 .
\end{aligned}
$$

We have chosen the minimum width $d$ so that five modes are allowed to propagate through the contact, $k_{\mathrm{F}} L=500$, and $k_{\mathrm{F}} l=1$. The latter choice needs a digression. The typical displacement $l$ of an EF in the course of thermalbath-induced fluctuations depends strongly on the microscopic nature of the EF. If the EF is produced by structural disorder in the vicinity of the channel, one can expect $l$ to be of the order of an interatomic distance, and $k_{\mathrm{F}} l \ll 1$. If the EF originates from electron hopping in the doped region of the structure, it is reasonable to expect that $l$ is of the order of the average distance between the impurities in the doped region. In this case the product $k_{\mathrm{F}} l$ can be as large as $1-10$ depending on size and shape of the structure. Another point to note is that a mechanical displacement of the scatterer is not the only reason for variations in the scattering phase $k_{\mathrm{F}} l$.
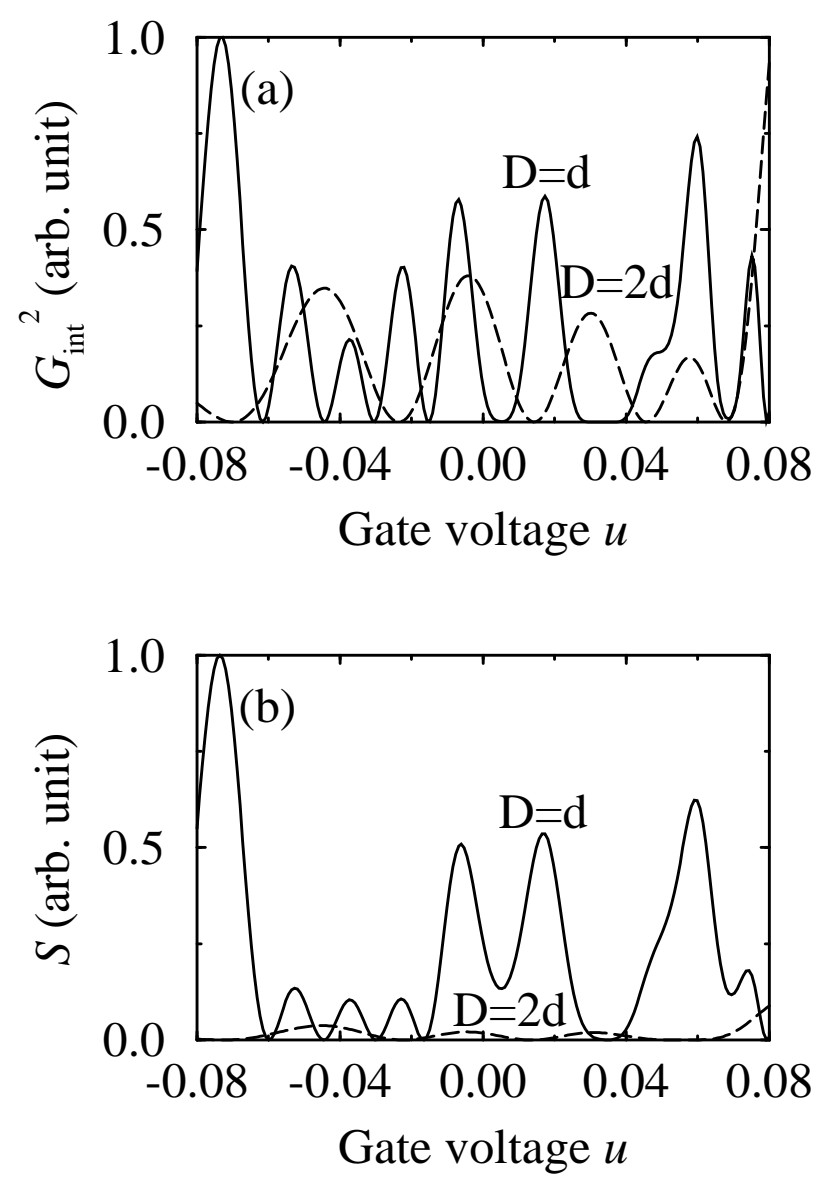

FIG. 1. Square of transconductance (panel $a$ ) and noise (panal $b$ ) as functions of dimensionless gate voltage parameter $u\left(V_{g}=u E_{\mathrm{F}}\right)$. Two different parabolic channels are used for which the relations between the maximum $(D)$ and minimum $(d)$ width are $D / d=1,2$. The lengths of the channels are in all cases $79.6 \lambda_{\mathrm{F}}$, where $\lambda_{\mathrm{F}}$ is the Fermi wavelength. The minimum width is chosen so as to let 5 modes propagate through the contact. The hopping distance $l$ for the fluctuator was taken to be $0.796 \lambda_{\mathrm{F}}$. The calculation was done for the source-drain voltage $V_{s d}=0.02 E_{\mathrm{F}}$ and for the temperature $T=10^{-3} T_{\mathrm{F}}, E_{\mathrm{F}}$ and $T_{\mathrm{F}}$ being the Fermi energy and temperature, respectively. 
Rearrangements in an extended defect containing several atoms can also cause variations in the scattering phase of order unity. Our phenomenological model does not allow for explicit calculations of the scattering phase, so we keep the simplest description of the mechanical displacement of the scatterer and use a reasonable value for the product $k_{\mathrm{F}} l$.

Taking the gate voltage parameter to be $u=0$ corresponds to considering the center of the plateaus in the $I-V_{g}$ curve. Our calculations were made for two different values of the ratio between the channel parameters, $D / d=1$ and 2 . The noise intensity is shown in Fig. 1 (panel $b$ ). As is clearly seen, there is a strong qualitative correlation between the noise and the square of the transconductance.

From our expressions (30) for noise and current, the similarity between the graphs seems plausible. Indeed, both quantities have oscillating parts of the form $\sin ^{2}\left(\sigma_{1,2}^{0}+\gamma\right)$. In addition, the expression for the noise contains another more slowly varying phase factor, $\sin ^{2}\left[w_{12}\left(E_{F}\right)\right]$, causing some modulation of the noise relative to the square of transconductance. The noise is weak in the regions $-0.06<u<-0.02$ for $D / d=1$ simply because this second phase factor causes almost every term to be small. For the case $D / d=2$ the phase itself is so small that the noise almost vanishes. Since there are many interference terms in the sum, some pairs of channels might lead to a stronger suppression than others.

In the final set of graphs, Fig. 2 (panels $a, b$, and $c$ ), we have studied how the current, transconductance and noise vary as functions of the applied source-drain voltage for the case of 10 propagating modes. Three different values of the dimensionless gate voltage parameter $u$ have been used, $u=-0.03(A), 0.0(B), 0.03(C)$. The range for which we are on the same conductance step in gate voltage, is approximately $-0.08<u<0.08$. The spread in the values of the transconductance for different values of $u$ is large for low-, and small and almost constant for high source-drain voltages. The reason for this is that, when the source-drain voltage is high, there is essentially an averaging over all interference patterns in the energy integrations above. In the absence of any bias at all, $V_{s d}=0$, the current is obviously zero, but also the noise (panel $c$ ) is zero. The expression for the noise (30) makes it apparent that this must always be the case.

Finally, we have found no simple relation between the magnitude of the noise and the position on the conductance plateau. The graphs for different values of $u$ may cross one another as is seen in panel $c$.

\section{DISCUSSION AND CONCLUSIONS}

Let us start the discussion by reviewing the results for a QPC with two current-carrying modes, Eq. (30)-(32). In this case we predict that the period of noise oscillations
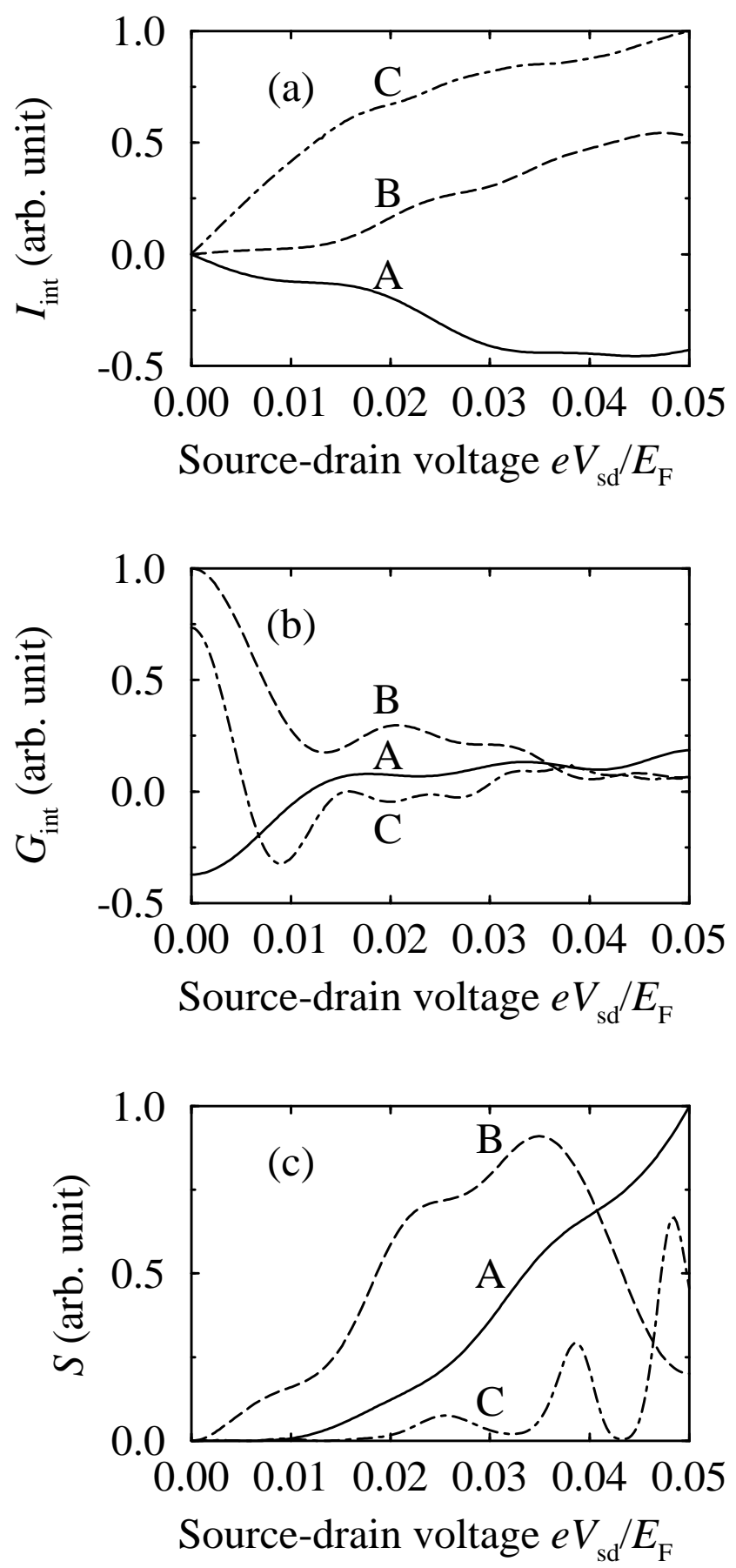

FIG. 2. Interference part of the current (panel $a$ ), transconductance (panel $b$ ) and noise (panel $c$ ) for a channel of constant width. They are here shown as functions of the source-drain voltage parameter $e V_{s d} / 2 E_{\mathrm{F}}$ for 3 different values of the dimensionless gate voltage $u\left(V_{g}=u E_{\mathrm{F}}\right)$. In $A$, $u=-0.03$ while in $B, u=0$ and in $C, u=0.03$. The lengths of the channels are in all cases $79.6 \lambda_{\mathrm{F}}$, where $\lambda_{\mathrm{F}}$ is the Fermi wavelength. The minimum width is chosen so that 10 modes propagate through the contact. The hopping distance $l$ for the fluctuator was taken to be $0.796 \lambda_{\mathrm{F}}$. The calculation was done for the temperature $T=10^{-3} T_{\mathrm{F}}, T_{\mathrm{F}}$ being the Fermi temperature. 
as a function of external parameters should be half the period of oscillations in the current. Verification of this statement is important in order to confirm (or falsify) our model.

In the general case when there is no restriction on the number of conducting modes we conclude the following.

- The dependence on temperature of the telegraph noise is determined by the factor $\cosh ^{-2}\left(\Delta / k_{\mathrm{B}} T\right)$. Comparing with experiments one can determine the interlevel spacing, $\Delta$, of the elementary fluctuator (EF). This would help to identify the nature of the EF.

- The width, $\Gamma$, of the Lorentzian that describes the frequency dependence of the noise, is also a function of temperature. In fact it reflects the most important dynamical property of the EF because the temperature dependence is different for quantum mechanical tunneling and activation (see Section III A).

- The main feature of our model is that variations in external parameters (such as $V_{g}, V_{s d}$ ) are assumed to only affect the phase functions $\sigma_{j}(E)$ (see Eq. (8)). The oscillatory behavior of the noise and the square of derivatives of the current (with respect to any external parameter) should then be qualitatively similar. In particular, the square of the transconductance is found to possess a striking similarity with the noise in the numerical simulation with five modes (see below).

- As functions of the source-drain voltage, any form of the interference current and noise might appear. It depends on the actual values of external parameters. Using different values of the gate voltage, even the sign of the current and transconductance can be changed. No simple relation between the noise and the gate voltage parameter has been found for the idealized case we have studied. The graphs may even cross one another. In other words, it seems to be irrelevant if we are close to a conduction step (in gate voltage), or at the center of a the plateau. Partly, this is a result of not letting the gate voltage vary enough so as to change the number of propagating modes.

From the numerical analysis with more than two propagating modes we conclude that there may be an observable correlation between the noise and the square of the transconductance. In essence, there is an additional phase factor $\sin \left(w_{i j}(E)\right)$ (see Eq. 29) in the noise. This may lead to favorable situations where the noise is suppressed while the transconductance is enhanced, leading to a large signal-to-noise ratio.

Finally, we would like to stress that, in spite of the idealized character of the adopted model, our conclusions seem rather general. Indeed, any kind of timefluctuations in the scattering phase shift by any scatterer should lead to a similar behavior. In that case the parameter $w_{i j}$ will have a different meaning and could in principle be of order unity.

\section{ACKNOWLEDGMENTS}

This work was supported by NorFA, grant no. 93.30.155, by the Nordic Research Network on the Physics of Nanometer Electronic Devices, and by the Swedish Natural Science Research Council (NFR). JPH also gratefully acknowledges the hospitality of the Department of Physics at Oslo University, Norway.

${ }^{1}$ L. I. Glazman and A. V. Khaetskii, Europhys. Lett. 9, 263 (1989)

${ }^{2}$ L. P. Kouwenhoven, B. J. van Wees, C. J. P. M. Harmans, J. G. Williamson, H. van Houten, C. J. J. Beenakker, C. T. Foxon, and J. J. Harris, Phys. Rev. B 39, 8040 (1989).

${ }^{3}$ A. M. Zagoskin, Pis'ma Zh. Eksp. Tepr. Fiz. 52, 1043 (1990) [JETP Lett. 52, 435 (1991)].

${ }^{4}$ N. K. Patel, J. T. Nichols, L. Martin-Moreno, M. Pepper, J. E. F. Frost, D. A. Ritchie, and G. A. C. Jones, Phys. Rev. B 44,13 549 (1991).

${ }^{5}$ C. J. J. Beenakker and H. van Houten, Solid State Physics 44, 1 (1991)

${ }^{6}$ R. Taborski, A. K. Geim, and P. E. Lindeløf, Superlattices and Microstructures 12, 137 (1992).

${ }^{7}$ R. Taborski, A. K. Geim, M. Persson, and P. E. Lindeløf, Phys. Rev. B. 49, 7813 (1994).

${ }^{8}$ A. Szafer and A. D. Stone, Phys. Rev. Lett. 62, 300 (1989).

${ }^{9}$ G. Kirczenow, Phys. Rev. B 39, 10452 (1989).

${ }^{10}$ A. M. Zagoskin, I. O. Kulik, Fiz. Nizk. Temp. 16, 911 (1990) [Sov. J. Low. Temp. Phys. 16, 533 (1990)].

${ }^{11}$ Hongqi Xu, Phys. Rev. B 47, 15630 (1993).

12 A. M. Zagoskin and R. I. Shekhter, Phys. Rev B50,15 August issue (1994).

${ }^{13}$ M. J. Laughton, J. R. Barker, J. A. Nixon, and J. H. Davies, Phys. Rev. B 1150 (1991).

${ }^{14}$ L. I. Glazman and M. Jonson, Phys. Rev. B 44, 3810 (1991).

${ }^{15}$ P. E. Lindel $\varnothing f$ (private communication).

${ }^{16}$ L. I. Glazman, G. B. Lesovik, D. E. Khmelnitskii, and R. I. Shekhter, Pis'ma Zh. Eksp. Teor. Fiz. 48, 218 (1988) [JETP Lett. 48, 238 (1988)].

17 A. Brataas, K. A. Chao, Mod. Phys. Lett. B 7, 1021 (1993).

${ }^{18}$ Yu. M. Galperin, V. G. Karpov, and V. I. Kozub, Adv. Phys. 38, 669 (1989).

${ }^{19}$ K. S. Ralls, W.J. Skocpol, L. D. Jackel, R. E. Howard, L. A. Fetter, R.W. Epworth, and D. M. Tennant, Phys. Rev. Lett. 52, 228 (1984).

${ }^{20}$ C. T. Rogers and R. A. Buhrman, Phys. Rev. Lett. 53, 1272 (1984). 
${ }^{21}$ K. S. Ralls and R. A. Buhrman, Phys. Rev. Lett 60, 2434 (1988).

${ }^{22}$ N. M. Zimmerman, B. Golding, and W. H. Haemmerle, Phys. Rev. Lett. 671322 (1991).

${ }^{23}$ B. Golding, N. Zimmerman, and S. N. Coppersmith, Phys. Rev. Lett. 68, 998 (1992).

${ }^{24}$ D. C. Ralph and R. A. Buhrman, Phys. Rev. Lett 69, 2118 (1992).

${ }^{25}$ P. W. Anderson, B. I. Halperin, and C. M. Varma, Philos. Mag. 25, 1 (1972).

${ }^{26}$ W.A. Phillips, J. Low Temp. Phys. 7, 351 (1972).

${ }^{27}$ V. G. Karpov, M. I. Klinger, and F. N. Ignat'ev, Solid State Commun. 44, 333 (1982); Sov. Phys. JETP 57, 439 (1983).

${ }^{28}$ C. W. Gardiner, Handbook of Stochastic Methods(Springer-Verlag, Berlin, Heidelberg, New York, Tokyo, 1985). 